\title{
Probing the opacity of local Universe GAMA galaxies using attenuation-inclination relations
}

\author{
Ellen Andrae ${ }^{1}$, Richard J. Tuffs ${ }^{1}$, Cristina C. Popescu ${ }^{2}$, \\ Mark Seibert ${ }^{3}$ and the GAMA Team \\ ${ }^{1}$ Max-Planck-Institut für Kernphysik, Heidelberg, Germany \\ ${ }^{2}$ Jeremiah Horrocks Institute for Astrophysics and Supercomputing, Preston, UK \\ ${ }^{3}$ Observatories of the Carnegie Institution of Washington, Pasadena, USA \\ email: Ellen.Andrae@mpi-hd.mpg.de
}

\begin{abstract}
Even though attenuation by dust strongly attenuates and reddens the UV/optical spectral energy distributions of spiral galaxies, quantifying these effects is challenging, particularly when, as is most often the case, infrared measurements of the absorbed energy are not available. We have initiated a study to model the dependence on inclination of the attenuation of light from galaxies measured in the Galaxy and Mass Assembly (GAMA) survey. We present preliminary results using SDSS galaxies in the phase-I GAMA catalogue, comparing observed attenuation-inclination relations with the predictions of the radiation transfer model of Popescu et al. (2011) to derive a statistical measurement of the mean face-on B-band optical depth of disks $\tau_{B}^{f}$ in a sample of spiral galaxies complete to the SDSS spectroscopic r-band limit of $17.8 \mathrm{mag}$.
\end{abstract}

Keywords. ISM: dust, extinction, galaxies: fundamental parameters, galaxies: ISM, galaxies: luminosity function, galaxies: statistics

\section{Introduction}

The presence of dust changes the spectral energy distributions (SEDs) of galaxies, as grains absorb optical / UV light, re-emitting in the infrared (primarily in the 50 - 300 $\mu \mathrm{m}$ far-IR range). This markedly changes the perceived SEDs in the UV-optical range, even for local Universe galaxies (e.g. Popescu \& Tuffs 2002; Driver et al. 2007). There are basically three methods for quantifying the attenuation by dust of the spatially integrated stellar emission from galaxies, as routinely measured in broad-band UV/optical surveys. The most direct method is to measure the absorbed UV/optical light in the farIR, and use a radiation transfer (RT) analysis of the combined UV/optical-FIR/submm SED to infer the attenuation of starlight as a function of wavelength in the UV/optical range. However, the limited sky coverage and depth of contemporary FIR/submm surveys effectively limit the application of this technique to incomplete subsets of the galaxy population. The second method is to use measurements of nebular emission lines with a known intrinsic luminosity ratio, such as hydrogen recombination lines, to probe the dust attenuating the broad-band emission. This is potentially a very powerful technique, but requires a careful treatment of the strong local attenuation by dust in star-formation regions and a combined spatial and spectral coverage of the whole galaxy, which is difficult to realise for large statistical samples. The third method is to statistically measure the dimming in integrated luminosity of starlight from dusty galaxies as the dust-bearing 

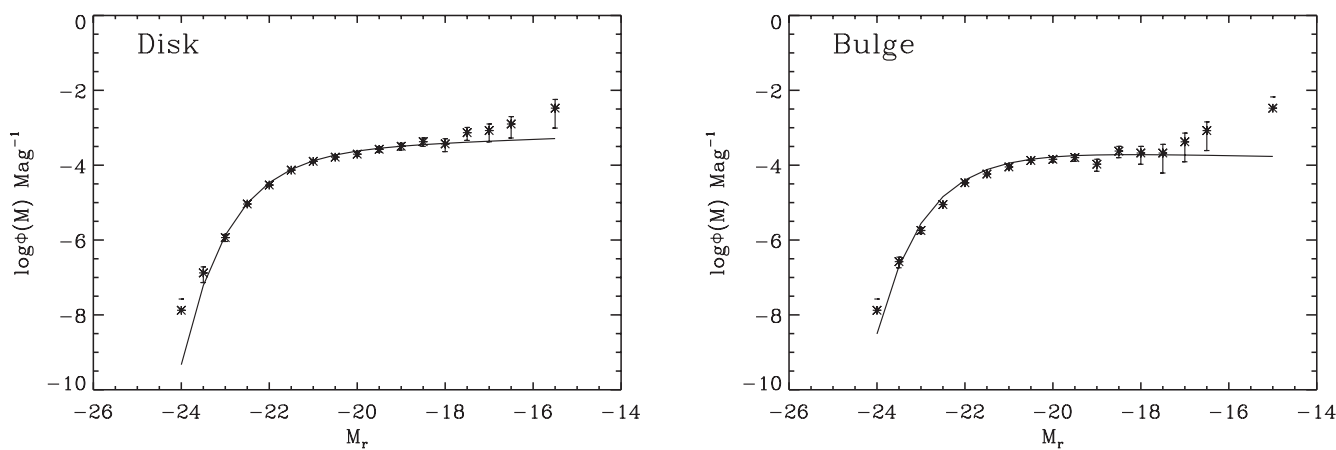

Figure 1. r-band luminosity distributions of the disks (left) and bulges (right) for inclinations $0.2<1-\cos (i)<0.3$. The curves indicate the fitted Schechter functions with the parameter $\alpha$ constrained from fits to disks and bulges for all spiral galaxies with $1-\cos (i)<0.3$ (see text).

disks are progressively observed from more edge-on viewpoints. We have embarked upon a project to model this so-called "attenuation-inclination" relation using the full wavelength range (UV to Near-IR) of the phase-I data on disk-dominated galaxies from the GAlaxy and Mass Assembly spectroscopic survey (GAMA; Driver et al. 2011). Here, we present preliminary results for bright $(\mathrm{r}<17.77)$ spiral galaxies in the SDSS.

\section{Attenuation-Inclination relation}

The basis for this work are galaxies with available bulge-disk decompositions and inclinations from Simard et al. (2011), with r-band magnitudes and redshifts taken from the GAMA catalogue. This results in a sample of $\sim 14 \mathrm{k}$ galaxies with $m_{r}<17.77$ mag. In deriving the attenuation-inclination relation, it is critical to take into account the different behaviour of bulges and disks. As the (dustless) bulge is more centrally placed in the disk, it is more strongly attenuated (e.g. Tuffs et al. 2004). We employ the approach by Driver et al. (2007) in which luminosity functions are fitted to subsamples of galaxies devided into inclination bins to find the turn-over magnitude, $M_{*}$, in a Schechter function as a function of inclination $i$ [i.e. $M_{*}$ versus $1-\cos (i)$ ]. This is an iterative process in which the mean face-on B-band optical depth of the disks $\tau_{B}^{f}$ is derived by fitting the observed attenuation-inclination relations with the RT model of Tuffs et al. (2004). This is done separately for disks and bulges, following the procedure outlined below. Iteration is required because the bulge-to-total luminosity ratios $(\mathrm{B} / \mathrm{T})$ should be based on intrinsic rather than apparent luminosity ratios.

(a) Apply the current best attenuation-inclination correction estimates of the disks and bulges (no correction applied for the first iteration).

(b) Recompute B/T. Apply B $/ \mathrm{T}<0.8$ cut (i.e. excluding elliptical galaxies).

(c) Derive the luminosity distribution (LD) using the 1/Vmax method for all disks and bulges with low inclination $[1-\cos (i)<0.3]$. Fit Schechter functions to the LDs in order to obtain the faint-end slope, $\alpha$, for low inclination.

(d) Derive the disk and bulge LDs for the full sample in equidistant $\cos (i)$ intervals. Fit Schechter functions with $\alpha$ fixed to the value from step (3). The final iteration example is shown in Fig. 1.

(e) Plot the recovered turnover magnitude $M_{*}$ versus $1-\cos (i)$. Determine the improved attenuation-inclination correction estimates by fitting the RT model (as a function of $\tau_{B}^{f}$ ) to the bulge and disk data (leaving out the incomplete interval $[0.9<1-\cos (i)<1])$. The final iteration example is shown in Fig. 2 .

$(f)$ Repeat $a-e$ until convergence. 

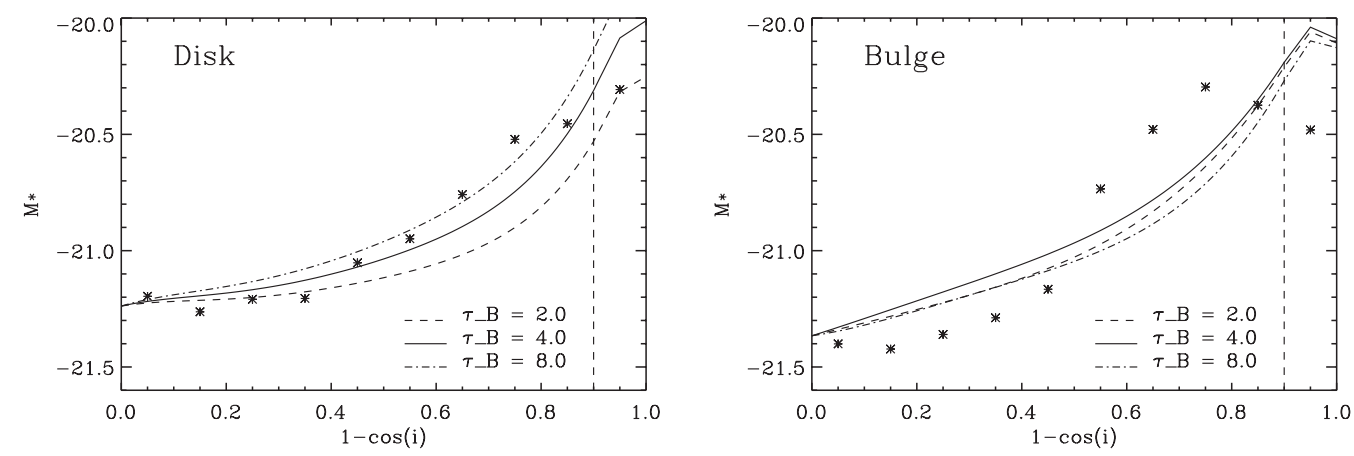

Figure 2. The r-band attenuation-inclination relation for disks (left) and bulges (right). The lines represent predicted relations derived using the model of Tuffs et al. (2004) for values of $\tau_{B}^{f}=2,4$, and 8 . The best fitting values are $\tau_{B, \text { disk }}=4.1$ and $\tau_{B, \text { bulge }}=4.0$.

The best fitting values for $\tau_{B}^{f}$ derived from the disk and bulge attenuation-inclination relations are $\tau_{B}^{f}=4.1$ and $\tau_{B}^{f}=4.0$, respectively. These results are consistent with the value $\tau_{B}^{f}=3.8 \pm 0.7$ found by Driver et al. (2007) using the B-band Millenium sample. As the inclination $1-\cos (i)$ is biased low (i.e. galaxies appear rounder than they are, due to resolution effects and the intrinsic thickness of the disk), high inclination galaxies with high attenuations appear at too low inclinations. Consequently, the derived values of $\tau_{B}^{f}$ may be considered as upper limits. While the best fitting RT model for the disk is nevertheless a good representation of the data, this bias becomes obvious for the bulge. Future higher resolution data will mitigate this effect as well as improve the bulge-disk decomposition.

\section{Outlook}

The attenuation-inclination relation will be derived for the full GAMA dataset from the FUV to the near-IR with its excellent statistics and spectroscopic depth (340k galaxies complete to $\mathrm{r} 19.8$ over ca. $320 \mathrm{sq}$ deg out to $\mathrm{z} \sim 0.4$ ). In addition, for a subset of galaxies measured by WISE and Herschel in the MIR/FIR/submm and with quantitative optical spectroscopy from GAMA it will be possible to test the self-consistency of the values for $\tau_{B}^{f}$ gained from the attenuation-inclination relation with those found from RT modelling of nebular emission lines and the broad-band UV-submm SED. If consistency can be achieved for a fixed geometry of stars and dust, the attenuation-inclination relation could be used to predict the face-on attenuation. The multicomponent morphological decompositions and quantitative spectroscopy of the GAMA dataset will ultimately allow separate analyses of different categories of rotationally-supported galaxies separated according to size, stellar mass, star-formation rate, star-formation history, morphology and environment.

\section{References}

Driver, S. P., Hill, D. T., Kelvin, L. S., et al. 2011, MNRAS, 413, 971

Driver, S. P., Popescu, C. C., Tuffs, R. J., et al. 2007, MNRAS, 379, 1022

Popescu, C. C. \& Tuffs, R. J. 2002, Reviews in Modern Astronomy, 15, 239

Popescu, C. C., Tuffs, R. J., Dopita, M. A., et al. 2011, AESA, 527, A109

Simard, L., Mendel, J. T., Patton, D. R., et al. 2011, ApJS, 196, 11

Tuffs, R. J., Popescu, C. C., Völk, H. J., et al. 2004, A\&广A, 419, 821 